\title{
A GENERALIZATION OF THE ARAMATA-BRAUER THEOREM
}

\author{
SANDRA L. RHOADES
}

(Communicated by Ronald M. Solomon)

\begin{abstract}
ABSTRACr. The Aramata-Brauer Theorem says that the regular character minus the principal character of a finite group can be written as a positive rational linear combination of induced linear characters. In the language of Artin $L$-series this says that $\zeta_{E}(s) / \zeta_{F}(s)$ is entire, where this is the quotient of the Dedekind $\zeta$-functions of a Galois extension $E / F$ of number fields. Given any subset of characters of a finite group, we will give a necessary and sufficient condition for when a character is a positive rational linear combination of characters from this specified subset. This result implies that the regular character plus or minus any irreducible character can be written as a positive rational linear combination of induced linear characters. This both generalizes and gives a new proof of the Aramata-Brauer Theorem.
\end{abstract}

\section{INTRODUCTION AND STATEMENT OF MAIN RESULTS}

The Aramata-Brauer Theorem says that the regular character minus the principal character of a finite group $G$ can be written as a positive rational linear combination of characters induced from nonprincipal, linear characters of cyclic subgroups of $G$. This character-theoretic result has important implications for the theory of Artin $L$-series. If $E / F$ is a Galois extension of algebraic number fields with Galois group $G$ and $\psi$ is any character of $G$, there is an Artin $L$-series, $L(s, \psi, E / F)$, which encodes some important arithmetic properties of this extension. Artin's Conjecture is that these $L$-series are always analytic at every complex point $s \neq 1$. Utilizing Brauer's Theorem that all Artin $L$-series are meromorphic, it can be shown that Artin's Conjecture is true for characters which can be written as a positive rational linear combination of characters induced from linear characters of subgroups of $G$. Furthermore, this is essentially the only general family of characters for which Artin's Conjecture is known to be true. Thus the Aramata-Brauer Theorem proves Artin's Conjecture for the $L$-series attached to the regular character minus the principal character of $G$ (this $L$-series is seen to be the quotient $\zeta_{E}(s) / \zeta_{F}(s)$ of Dedekind $\zeta$-functions of $E / F)$.

In [7, p. 871] Heilbronn couched Artin's Conjecture in the language of character theory. Specifically, for a fixed point $s_{0} \in \mathbb{C}-\{1\}$, he defined the virtual

Received by the editors February 11, 1992.

1991 Mathematics Subject Classification. Primary 20C15; Secondary 11M41.

Research partially supported by NSA grant MSPF-038-90. 
character

$$
\theta_{G}=\sum_{\psi \in \operatorname{Irr}(G)} n_{\psi} \psi,
$$

where $\operatorname{Irr}(G)$ is the set of all irreducible characters of $G$ and $n_{\psi}$ is the order of the zero or pole of the meromorphic Artin $L$-series $L(s, \psi, E / F)$ at $s_{0}$. The connection between these virtual characters $\theta_{G}$ and Artin's Conjecture rests on the observation that $\theta_{G}$ is a character if and only if all Artin $L$-series attached to the extension $E / F$ are analytic at $s_{0}$. Moreover, if $\psi$ is any character of $G$, it follows from basic properties of $L$-series that the order of the zero or pole of $L(s, \psi, E / F)$ at $s_{0}$ is equal to the inner product $\left\langle\theta_{G}, \psi\right\rangle$. In particular, $L(s, \psi, E / F)$ is analytic at $s_{0}$ if and only if this inner product is nonnegative. Since, as noted above, $L\left(s, \lambda^{*}, E / F\right)$ is analytic at $s_{0}$ for any linear character $\lambda$ of any subgroup of $G$ (where a star will always denote induction from a character of a subgroup to a character of $G$ ), we have that

\section{(*) $\left\langle\theta_{G}, \lambda^{*}\right\rangle \geq 0$ for all linear characters $\lambda$ of all subgroups of $G$.}

Heilbronn's ideas were extended by Stark [8], Foote-Murty [5], Foote [4], and Foote-Wales [6] to produce results of the following form: By imposing conditions on $G$, such as solvability, and by setting a bound on the order of the zero or pole of $\zeta_{E}(s)$ at $s_{0}$, they were able to prove that all Artin $L$-series attached to the extension $E / F$ are analytic at $s_{0}$.

In this paper, we consider the family $\mathscr{H}$ of all "candidates" for the numbertheoretically defined virtual characters $\theta_{G}$ by taking property $(*)$ above as the defining characteristic for any virtual character $\theta_{G}$ to be a member of $\mathscr{H}$. This specifies the family in purely character-theoretic terms for any finite group $G$. Our main result is that any character of $G$ whose inner product with every element of $\mathscr{H}$ is nonnegative must be a positive rational linear combination of induced linear characters. We thus see that the methods for proving Artin's Conjecture in the references cited in the preceding paragraph are essentially equivalent to the general method of proving that certain characters are positive rational linear combinations of induced linear characters. As an application of our main result we show that the regular character plus or minus any irreducible character can be written as a positive rational linear combination of linear characters induced from cyclic subgroups. In particular, this both generalizes and gives a new proof of the Aramata-Brauer Theorem.

In fact, for our character-theoretic results we need not restrict to induced linear characters. The analogous result holds when we use any subset of the characters of our finite group in place of the set of induced linear characters. Given a subset of characters of the group we give a necessary and sufficient condition for when a character can be written as a positive rational linear combination of characters from this subset.

Let $\mathscr{F}$ be a subset of the characters of a finite group $G$. For instance $\mathscr{F}$ might consist of all the induced linear characters of $G$. A virtual character $\theta$ of $G$ will be referred to as a Heilbronn character with respect to $\mathscr{F}$ if the inner product of $\theta$ and $\phi$ is nonnegative for all $\phi \in \mathscr{F}$. Let $\mathscr{H}(\mathscr{F})$ denote the set of all Heilbronn characters with respect to $\mathscr{F}$, where $\mathscr{H}(\varnothing)$ is the set of all virtual characters of $G$. The phrase Heilbronn character will be used in place of Heilbronn character with respect to $\mathscr{F}$ when this does not lead to confusion. Note that, in general, a Heilbronn character need not be a character. 
On the other hand every character is a Heilbronn character for every set $\mathscr{F}$ as the inner product of two characters is always nonnegative.

Theorem 1. Let $\mathscr{F}$ be a subset of characters of some finite group $G$, and let $\psi$ be a nonzero virtual character of $G$. Then $\psi$ can be written as a positive rational linear combination of characters from $\mathscr{F}$ if and only if the inner product of $\psi$ with every Heilbronn character with respect to $\mathscr{F}$ is nonnegative.

An easy consequence of this theorem is a generalization of the classical Aramata-Brauer Theorem (first proved in [1-3]):

Theorem 2. Let $\rho$ be the regular character of $G$ and $\chi$ be any irreducible character of $G$. Then there exist positive rational numbers $s_{i}$ and linear characters $\lambda_{i}$ of cyclic subgroups of $G$ such that $\rho-\chi=\sum_{i} s_{i} \lambda_{i}^{*}$. Similarly $\rho+\chi$ is a positive rational linear combination of linear characters induced from cyclic subgroups of $G$.

Corollary (Aramata-Brauer). Let $\rho$ be the regular character and $1_{G}$ the principal character of $G$. There exist $s_{i} \in \mathbb{Q}^{+}$and nonprincipal linear characters $\lambda_{i}$ of cyclic subgroups of $G$ such that $\rho-1_{G}=\sum_{i} s_{i} \lambda_{i}^{*}$.

The following proposition is not used in the proofs of our theorems. But we include it because with only mild restrictions on $\mathscr{F}$ it ensures the existence of Heilbronn characters with respect to $\mathscr{F}$ which are not characters of $G$. In addition, the proof of this result indicates how to construct these Heilbronn characters.

Proposition 3. If no positive integer multiple of the irreducible character $\chi_{j}$ belongs to $\mathscr{F}$, then there exists a character $\theta$ of $G$ orthogonal to $\chi_{j}$ such that $\theta-\chi_{j}$ is a Heilbronn character with respect to $\mathscr{F}$.

\section{PRELIMINARY RESULTS}

The relation between Heilbronn characters and characters of $G$ that can be written as a positive rational linear combination of characters in $\mathscr{F}$ is captured in Lemma 1 (which is an elementary result about vector spaces). A second lemma is needed to ensure that we have rational instead of real coefficients. The proofs of the main results follow directly from these two lemmas.

Definition. Let $\langle$,$\rangle denote the usual inner product on \mathbb{R}^{k}$, and let $F$ be any nonempty subset of $\mathbb{R}^{k}$. Define

(1) $\mathscr{H}(F)=\left\{x \in \mathbb{R}^{k} \mid\langle f, x\rangle \geq 0\right.$ for all $\left.f \in F\right\}$ and

(2) $\mathscr{C}(F)=\left\{\sum_{i=1}^{n} w_{i} f_{i} \mid n \in \mathbb{Z}^{+}, f_{i} \in F\right.$ and $w_{i} \in \mathbb{R}^{+}$for all $\left.i\right\}$.

It is clear that $\mathscr{H}(F)$ is the set of all vectors in $\mathbb{R}^{k}$ which are either acute or orthogonal to all vectors in $F$. Note that the use of the notation $\mathscr{H}(F)$ is consistent with the use of the notation $\mathscr{H}(\mathscr{F})$ for Heilbronn characters with respect to $\mathscr{F}$ when each vector $\left(r_{1}, r_{2}, \ldots, r_{k}\right) \in \mathbb{R}^{k}$ is identified with the virtual character $\sum r_{i} \chi_{i}$ of $G$. To tie these two definitions into the theory of cones, we note that $\mathscr{H}(F)$ is the polar cone of $-F$ and $\mathscr{C}(F)$ is the conical hull of $F$. It should also be noted that Lemma 1 can be deduced from the results in the theory of cones (see, e.g., [9, pp. 51-56]), but it is important to give a self-contained proof for this paper. 
Lemma 1. Let $F$ be a nonempty finite subset of $\mathbb{R}^{k}$ not containing zero. Assume that all the elements of $F$ have nonnegative coordinates. Then $\mathscr{H}(\mathscr{H}(F))=$ $\mathscr{C}(F)$.

Proof. We will first show that $\mathscr{C}(F) \subseteq \mathscr{H}(\mathscr{H}(F))$. Let $n$ be in the conical hull of $F$. Then there exist nonnegative real numbers $a_{i}$ and elements $f_{i}$ of $F$ such that $n=\sum a_{i} f_{i}$. To show $n \in \mathscr{H}(\mathscr{H}(F))$ it suffices to show that $\langle n, x\rangle \geq 0$ for all $x \in \mathscr{H}(F)$. Let $x \in \mathscr{H}(F)$. Then $\left\langle f_{i}, x\right\rangle \geq 0$ for all $i$. So $\langle n, x\rangle=\left\langle\sum a_{i} f_{i}, x\right\rangle=\sum a_{i}\left\langle f_{i}, x\right\rangle \geq 0$. Thus $\mathscr{C}(F) \subseteq \mathscr{H}(\mathscr{H}(F))$.

Now to show that $\mathscr{H}(\mathscr{H}(F)) \subseteq \mathscr{C}(F)$, let $n \in \mathscr{H}(\mathscr{H}(F))$ and suppose that $n$ is not an element of $\mathscr{C}(F)$. Let

$$
O_{n}=\left\{x \in \mathbb{R}^{k} \mid\langle n, x\rangle<0\right\} .
$$

We claim it suffices to show that $\mathscr{H}(F) \cap O_{n} \neq \varnothing$ : Suppose $\mathscr{H}(F) \cap O_{n}$ is nonempty. Then there exists an element $z \in \mathscr{H}(F)$ such that $\langle n, z\rangle<0$, but this contradicts the fact that $n$ is an element of $\mathscr{H}(\mathscr{H}(F))$. So it remains to show that $\mathscr{H}(F) \cap O_{n} \neq \varnothing$.

Let $d: \mathscr{C}(F) \rightarrow \mathbb{R}$ be the Euclidean distance from any point in $\mathscr{C}(F)$ to $n$. As $d$ is a distance function and the conical hull of $F$ a closed set, $d$ must achieve a minimum at some point $m$ in $\mathscr{C}(F)$. Thus $d(m) \leq d(a)$ for all $a \in \mathscr{C}(F)$. By assumption $n$ is not an element of $\mathscr{C}(F)$, so $m \neq n$. We define a vector $c$ which is in the same plane as $m$ and $n$, and perpendicular to $m+n$ by setting

$$
c=\langle n, m+n\rangle m-\langle m, m+n\rangle n .
$$

We claim that $c \in \mathscr{H}(F) \cap O_{n}$.

To show this we will use inequality arguments involving division by inner products; so first let us establish that all inner products involved will be positive. It will be sufficient to know that $m$ and $n$ have nonnegative coordinates. As $m \in \mathscr{C}(F), m$ must have nonnegative coordinates. As for $n$, suppose without loss of generality that its first coordinate $n_{1}$ is negative. Define $x=(1,0, \ldots, 0)$. As all the elements of $F$ have nonnegative coordinates, $x \in \mathscr{H}(F)$. But $\langle n, x\rangle=n_{1}<0$ and $n \in \mathscr{H}(\mathscr{H}(F))$, so we have a contradiction. Thus $n$ must have nonnegative coordinates.

Let $f \in F$. To show that $c \in \mathscr{H}(F)$ we need to show that $\langle c, f\rangle>0$, or equivalently that $\langle n, m+n\rangle\langle m, f\rangle-\langle m, m+n\rangle\langle n, f\rangle>0$. This will follow from the as yet unestablished inequalities $\langle m, m+n\rangle /\langle n, m+n\rangle<1$ and $1 \leq\langle m, f\rangle /\langle n, f\rangle$.

We will begin by establishing the first of these two inequalities. As $m$ was chosen to be the point in the cone $\mathscr{C}(F)$ of minimum distance to $n$ and $\mathscr{C}(F)$ is closed under positive multiples, we can deduce that $\langle n-m, m\rangle=0$. Thus by the Pythagorean Theorem we have $\|n\|^{2}=\|m\|^{2}+\|n-m\|^{2}$, which implies that $\|m\|^{2}<\|n\|^{2}$. This implies that $\langle m, m\rangle+\langle m, n\rangle\langle\langle n, m\rangle+\langle n, n\rangle$. By the linearity of the inner product, this becomes $\langle m, m+n\rangle /\langle n, m+n\rangle<1$.

The second inequality will follow from $\langle n-m, f\rangle \leq 0$, which we will establish using the minimality of $m$. Proceeding by way of contradiction, suppose $\langle n-m, f\rangle>0$, and define $x=\gamma f+m$ where $\gamma=\langle n-m, f\rangle /\|f\|^{2}$. Now $\gamma<2\langle n-m, f\rangle /\|f\|^{2}$. Since $\gamma$ is positive, this inequality yields the new inequality $\gamma^{2}\|f\|^{2}+2 \gamma\langle f, m-n\rangle<0$. Adding $\|m-n\|^{2}$ to both sides of this inequality gives $\|\gamma f+m-n\|^{2}<\|m-n\|^{2}$. This in turn implies that 
$\|x-n\|<\|m-n\|$ or $d(x)<d(m)$. But $f$ and $m$ are in the linear cone $\mathscr{C}(F)$, so $x$ is an element of $C$, a contradiction. Therefore $\langle n-m, f\rangle \leq 0$. As this was the remaining inequality needed, we have shown that $c$ is an element of $\mathscr{H}(F)$.

It remains to show that $c \in O_{n}$. To do so we need to show that

$$
\langle c, n\rangle=\langle\langle n, m+n\rangle m-\langle m, m+n\rangle n, n\rangle<0 .
$$

By the Cauchy-Bunyakovskii-Schwarz Inequality we have

$$
\langle m, n\rangle^{2}<\langle m, m\rangle\langle n, n\rangle,
$$

which implies that

$$
\langle m, n\rangle^{2}+\langle n, n\rangle\langle m, n\rangle-\langle m, m\rangle\langle n, n\rangle-\langle m, n\rangle\langle n, n\rangle<0 .
$$

This leads to

$$
\langle n, m+n\rangle\langle m, n\rangle-\langle m, m+n\rangle\langle n, n\rangle<0,
$$

and so we have

$$
\langle\langle n, m+n\rangle m-\langle m, m+n\rangle n, n\rangle<0,
$$

i.e., $\langle c, n\rangle<0$.

We have established that $c \in \mathscr{H}(F) \cap O_{n}$; therefore, $\mathscr{H}(F) \cap O_{n} \neq \varnothing$ and the proof of Lemma 1 is complete.

Lemma 2. Let $v_{1}, \ldots, v_{s}$ and $n$ be vectors in $\mathbb{R}^{k}$ with nonnegative rational coordinates. If $n$ can be written as a nonnegative real linear combination of $v_{1}, \ldots, v_{s}$, then $n$ can also be written as a nonnegative rational linear combination of $v_{1}, \ldots, v_{s}$.

Proof. By assumption there exist nonnegative real numbers $\alpha_{i}$ such that $n=$ $\alpha_{1} v_{1}+\cdots+\alpha_{s} v_{s}$. We are only concerned with the $\alpha_{i}$ which are nonzero, so if necessary replace $\left\{v_{1}, \ldots, v_{s}\right\}$ by one of its subsets and renumber so that $n=\alpha_{1} v_{1}+\cdots+\alpha_{r} v_{r}$ with each $\alpha_{i}$ a positive real number. Now we will renumber once again, if necessary, so that $\left\{v_{1}, \ldots, v_{p}\right\}$ is a maximal independent subset of $\left\{v_{1}, \ldots, v_{r}\right\}$.

Let $A$ be the $k \times r$ matrix with rational coefficients whose columns are the column vectors $v_{1}, \ldots, v_{r}$. Let $C$ be the column vector $n$, and let $X$ be the $r$-dimensional column vector whose coordinates are variables $x_{1}, \ldots, x_{r}$.

If $\left\{v_{1}, \ldots, v_{r}\right\}$ is an independent set of vectors (i.e., if $p=r$ ), then the system of linear equations given by $A X=C$ has a unique solution which is found by row-reducing the augmented matrix $(A \mid C)$ over its field of coefficients. This forces the solution to lie in the field $\mathbb{Q}$ of coefficients of $(A \mid C)$. Therefore, in this case, our given solution $\left(\alpha_{1}, \ldots, \alpha_{r}\right)$ which consists of positive real numbers must be the unique solution and so must consist of positive rational numbers. Thus, in this case, $n$ may be written as a nonnegative rational linear combination of $v_{1}, \ldots, v_{s}$ as was desired.

Now we are left to examine the case where the independent set $\left\{v_{1}, \ldots, v_{p}\right\}$ does not equal the full set $\left\{v_{1}, \ldots, v_{r}\right\}$ (i.e, $p<r$ ). Recall that if $A X=C$ is a consistent system of linear equations in $r$ unknowns having rank $p$, then all solutions $\left(x_{1}, \ldots, x_{r}\right)$ of this system can be obtained as follows: We may 
arbitrarily set $\left(x_{p+1}, \ldots, x_{r}\right)=\left(t_{1}, \ldots, t_{r-p}\right)$ and in doing so force each $x_{i}$ for $i=1, \ldots, p$ to be of the form

$$
x_{i}=b_{i 1} t_{1}+\cdots+b_{i r-p} t_{r-p}+d_{i}
$$

with $b_{i j}, d_{i}$ in the field of coefficients of $A X=C$ for all $i \in\{1, \ldots, p\}$ and all $j \in\{1, \ldots, r-p\}$. Note that $b_{i j}$ and $d_{i}$ are not dependent on the choice of $\left(t_{1}, \ldots, t_{r-p}\right)$. By this fact there exists a solution $\left(\alpha_{1}^{\prime}, \ldots, \alpha_{r}^{\prime}\right)$ so that $\alpha_{p+1}^{\prime}, \ldots, \alpha_{r}^{\prime}$ are positive rationals and are chosen close enough to $\alpha_{p+1}, \ldots, \alpha_{r}$ respectively to ensure that $\alpha_{1}^{\prime}, \ldots, \alpha_{p}^{\prime}$ are still positive real numbers. But now $\alpha_{1}^{\prime}, \ldots, \alpha_{p}^{\prime}$ are rational since $\alpha_{p+1}^{\prime}, \ldots, \alpha_{r}^{\prime}$ and $b_{i j}, d_{i}$ for all $i$ and $j$ are all rational. In other words $\left(\alpha_{1}^{\prime}, \ldots, \alpha_{r}^{\prime}\right)$ is a positive rational solution to $A X=$ $C$. This says that $n$ can be written as a nonnegative rational linear combination of $v_{1}, \ldots, v_{s}$, and completes the proof of this lemma.

\section{Proof OF THEOREM 1}

Let $\mathscr{F}$ and $\psi$ be as given in the statement of Theorem 1 . Now identify each virtual character $\sum r_{i} \chi_{i}$ of $G$ with the corresponding vector $\left(r_{1}, r_{2}, \ldots, r_{k}\right) \in$ $\mathbb{R}^{k}$. Viewing $\psi$ as a vector in $\mathbb{R}^{k}$, we note that the inner product of $\psi$ with each Heilbronn character with respect to $\mathscr{F}$ is nonnegative exactly when $\psi \in$ $\mathscr{H}(\mathscr{H}(\mathscr{F}))$. With this in mind we can easily prove Theorem 1. Suppose $\psi$ can be written as a positive rational linear combination of characters of $\mathscr{F}$. Then $\psi \in \mathscr{C}(\mathscr{F})$, but by Lemma $1 \mathscr{C}(\mathscr{F}) \subseteq \mathscr{H}(\mathscr{H}(\mathscr{F}))$, so the inner product of $\psi$ with each element of $\mathscr{H}(\mathscr{F})$ is nonnegative. This completes one direction of the proof.

For the other direction, suppose that the inner product of $\psi$ with each Heilbronn character with respect to $\mathscr{F}$ is nonnegative. Then $\psi \in \mathscr{H}(\mathscr{H}(\mathscr{F}))$ which by Lemma 1 is contained in $\mathscr{C}(\mathscr{F})$; thus, $\psi$ can be written as a positive real linear combination of characters in $\mathscr{F}$. Note that $\psi$ is actually a character of $G$ as $\mathscr{H}(\mathscr{F})$ contains all irreducible characters of $G$. Thus we may use Lemma 2 to deduce that $\psi$ can be written as a positive rational linear combination of characters of $\mathscr{F}$. This completes the proof of Theorem 1.

\section{ProOF OF THEOREM 2 AND ITS COROLLARY}

Define $\mathscr{L}$ to be the set of all characters of $G$ induced from linear characters of cyclic subgroups of $G$. We begin with a result on the magnitude of the coefficients of Heilbronn characters with respect to $\mathscr{L}$. Recall that $\rho$ is the regular character of $G$. If $\theta$ is a Heilbronn character with respect to $\mathscr{L}$, then as $\rho \in \mathscr{L}$ we know that $\langle\rho, \theta\rangle$, and thus $\theta(1)$, is nonnegative.

Proposition 4. Let $\theta$ be a Heilbronn character with respect to $\mathscr{L}$. Then $\theta=$ $\sum_{i=1}^{r} m_{i} \chi_{i}$ where $\left|m_{i}\right| \leq \theta(1)$ for all $i$.

Proof. Let $x$ be an element of $G$ and let $\sigma$ be an irreducible character of $\langle x\rangle$. By Frobenius Reciprocity we have $\left\langle\left.\theta\right|_{\langle x\rangle}, \sigma\right\rangle=\left\langle\theta, \sigma^{*}\right\rangle$. Now $\sigma^{*} \in \mathscr{L}$ implies that $\left\langle\theta, \sigma^{*}\right\rangle$ is nonnegative. Thus $\left\langle\left.\theta\right|_{\langle x\rangle}, \sigma\right\rangle \geq 0$ for all irreducible characters $\sigma$ of $\langle x\rangle$, so $\left.\theta\right|_{\langle x\rangle}$ is a character of $\langle x\rangle$. As $\left.\theta\right|_{\langle x\rangle}(x)$ is a sum of $\left.\theta\right|_{\langle x\rangle}(1)$ roots of unity we have $|\theta(x)| \leq \theta(1)$. Since $x$ was arbitrary we have

$$
\langle\theta, \theta\rangle=\frac{1}{|G|} \sum_{x \in G} \theta(x) \overline{\theta(x)}=\frac{1}{|G|} \sum_{x \in G}|\theta(x)|^{2} \leq \frac{1}{|G|} \sum_{x \in G} \theta(1)^{2}=\theta(1)^{2} .
$$


As $\theta$ is a Heilbronn character, there exist integers $m_{i}$ such that $\theta=\sum_{i=1}^{r} m_{i} \chi_{i}$. Noting that $\langle\theta, \theta\rangle=\sum_{i=1}^{r} m_{i}^{2}$, the above inequality becomes $\sum_{i=1}^{r} m_{i}^{2} \leq$ $\theta(1)^{2}$. This gives our desired conclusion $\left|m_{i}\right| \leq \theta(1)$ for all $i$.

Proof of Theorem 2. Let $\chi_{j}$ be an irreducible character of $G$. By Theorem 1 it suffices to show that $\left\langle\rho \pm \chi_{j}, \theta\right\rangle \geq 0$ for all Heilbronn characters $\theta$ with respect to $\mathscr{L}$. As $\left\langle\rho \pm \chi_{j}, \theta\right\rangle=\theta(1) \pm\left\langle\chi_{j}, \theta\right\rangle$, it suffices to show that $\left|\left\langle\chi_{j}, \theta\right\rangle\right| \leq \theta(1)$. But this follows immediately from the above proposition, so the proof is complete.

Proof of the corollary. By Theorem 2 there exist positive rational numbers $s_{i}$ and linear characters $\lambda_{i}$ of cyclic subgroups of $G$ such that $\rho-1_{G}=\sum_{i=1}^{r} s_{i} \lambda_{i}^{*}$. By Frobenius Reciprocity, $1_{G}$ is a constituent of any induced principal character. Thus if one of the $\lambda_{i}$ is a principal character, then we have $\left\langle 1_{G}, \sum_{i=1}^{r} s_{i} \lambda_{i}^{*}\right\rangle$ $\neq 0$. But $\left\langle 1_{G}, \rho-1_{G}\right\rangle=0$, so this is a contradiction. Thus none of the $\lambda_{i}$ are principal characters, and the corollary is established.

\section{Proof of Proposition 3}

The proof of Proposition 3 relies on the following lemma for which we need a few definitions. For any virtual character $\alpha$ of $G$ define the supporting indices of $\alpha$ to be the set $\left\{i \mid\left\langle\alpha, \chi_{i}\right\rangle \neq 0\right\}$. We will denote the supporting indices of $\alpha$ by $\operatorname{supp}(\alpha)$. Let $\mathscr{F}$ be any collection of characters of $G$ and let $I \subseteq\{1,2, \ldots, r\}$. Define $\mathscr{F}_{I}=\{\phi \in \mathscr{F} \mid \operatorname{supp}(\phi) \subseteq I\}$.

Lemma 3. Let I be a subset of $\{1,2, \ldots, r\}$. If $\theta_{0}$ is a Heilbronn character with respect to $\mathscr{F}_{I}$ and $\operatorname{supp}\left(\theta_{0}\right) \subseteq I$, then there exists $M \in \mathbb{Z}^{+}$such that $\theta_{0}+M\left(\sum_{i \notin I} \chi_{i}\right) \in \mathscr{H}(\mathscr{F})$.

Proof. If $I=\varnothing$ or $I=\{1,2, \ldots, r\}$ there is nothing to prove, so assume this is not the case. Let $\sigma=\sum_{i \notin I} \chi_{i}$. Choose $M \in \mathbb{Z}^{+}$such that $-M \leq\left\langle\theta_{0}, \phi\right\rangle$ for all $\phi \in \mathscr{F} \backslash \mathscr{F}$. To show $\theta_{0}+M \sigma \in \mathscr{H}(\mathscr{F})$, we need to show that

$$
\left\langle\theta_{0}+M \sigma, \phi\right\rangle \geq 0 \text { for all } \phi \in \mathscr{F} \text {. }
$$

If $\phi \in \mathscr{F}_{I}$, then $\operatorname{supp}(\phi) \subseteq I$, so $\langle\sigma, \phi\rangle=0$. Therefore we have

$$
\left\langle\theta_{0}+M \sigma, \phi\right\rangle=\left\langle\theta_{0}, \phi\right\rangle+M\langle\sigma, \phi\rangle=\left\langle\theta_{0}, \phi\right\rangle \text {. }
$$

As $\theta_{0}$ is assumed to be a Heilbronn character with respect to $\mathscr{F}_{I}$, we have $\left\langle\theta_{0}, \phi\right\rangle \geq 0$. Thus $\left\langle\theta_{0}+M \sigma, \phi\right\rangle \geq 0$ in this case.

If, on the other hand, $\phi \in \mathscr{F} \backslash \mathscr{F}_{I}$, then $\operatorname{supp}(\phi) \nsubseteq I$, so $\langle\sigma, \phi\rangle \geq 1$. Therefore $-M\langle\sigma, \phi\rangle \leq-M$. Since by choice of $M$ we have $-M \leq\left\langle\theta_{0}, \phi\right\rangle$, we get

$$
-M\langle\sigma, \phi\rangle \leq\left\langle\theta_{0}, \phi\right\rangle
$$

This implies that $\left\langle\theta_{0}+M \sigma, \phi\right\rangle \geq 0$ and completes the proof of Lemma 3 .

Proof of Proposition 3. Note $\mathscr{F}_{\{j\}}=\{\phi \in \mathscr{F} \mid \operatorname{supp}(\phi) \subseteq\{j\}\}=\{\phi \in \mathscr{F} \mid \phi=$ $m \chi_{j}$ for some $\left.m \in \mathbb{Z}^{+}\right\}$. Thus by our hypothesis we have $\mathscr{F}_{\{j\}}=\varnothing$. Therefore any virtual character of $G$ is a Heilbronn character with respect to $\mathscr{F}_{\{j\}}$; so, in particular, $-\chi_{j} \in \mathscr{H}\left(\mathscr{F}_{\{j\}}\right)$. The hypotheses of Lemma 3 are satisfied with $\theta_{0}=-\chi_{j}$, and so there exists $M \in \mathbb{Z}^{+}$such that $M\left(\sum_{i \neq j} \chi_{i}\right)-\chi_{j} \in \mathscr{H}(\mathscr{F})$. Now taking $\theta=\sum_{i \neq j} M \chi_{i}$ it is clear that $\theta$ is orthogonal to $\chi_{j}$, and we have the desired result. 


\section{ACKNOWLEDGMENT}

The author thanks the Department of Mathematics at the University of Vermont for its hospitality during her stay there, and also wishes to thank R. Foote for his warmth and inspiration without which this paper would not have been written.

\section{REFERENCES}

1. H. Aramata, Über die Teilbarkeit der Dedekindschen Zetafunktionen, Proc. Imp. Acad. Tokyo 7 (1931), 334-336.

2. _ Über die Teilbarkeit der Dedekindschen Zetafunktionen, Proc. Imp. Acad. Tokyo 9 (1933), 31-34.

3. R. Brauer, On the zeta-functions of algebraic number fields, Amer. J. Math. 69 (1947), 243-250.

4. R. Foote, Nonmonomial characters and Artin's conjecture, Trans. Amer. Math. Soc. 321 (1990), 261-272.

5. R. Foote and V. K. Murty, Zeros and poles of Artin L-series, Math. Proc. Cambridge Philos. Soc. 105 (1989), 5-11.

6. R. Foote and D. Wales, Zeros of order 2 of Dedekind zeta-functions and Artin's conjecture, J. Algebra 131 (1990), 226-257.

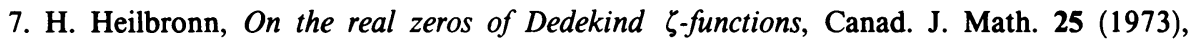
870-873.

8. H. Stark, Some effective cases of the Brauer-Siegel Theorem, Invent. Math. 23 (1974), 135-152.

9. J. Stoer and C. Witzgall, Convexity and optimization in finite dimensions. I, SpringerVerlag, Berlin, 1970.

Department of Mathematics, University of Massachusetts, AmHerst, Massachusetts 01003

E-mail address: rhoades@math.umass.edu 